OPEN ACCESS

Edited by:

Alec Schmaier,

Beth Israel Deaconess Medical Center and Harvard Medical School,

United States

Reviewed by:

Pavan Bendapudi,

Beth Israel Deaconess Medical Center and Harvard Medical School,

United States

Bipin P. Kulkarni,

Indian Council of Medical

Research, India

*Correspondence:

Amanda Fernández-Rodríguez amandafr@isciii.es

Salvador Resino

sresino@isciii.es

tThese authors have contributed equally to this work

Specialty section:

This article was submitted to Hematology,

a section of the journal

Frontiers in Medicine

Received: 31 May 2021 Accepted: 03 September 2021 Published: 30 September 2021

Citation:

Ceballos FC, Ryan P, Blancas $R$, Martin-Vicente M, Vidal-Alcántara EJ, Peréz-García F, Bartolomé S,

Churruca-Sarasqueta J, Virseda-Berdices A

Martínez-González O,

Brochado-Kith O, Rava M, Vilches-Medkouri C, Blanca-López N,

Ramirez Martinez-Acitores I, Moreira-Escriche P, De Juan C, Resino S, Fernández-Rodríquez $A$ and Jiménez-Sousa MÁ (2021) Are Reduced Levels of Coagulation Proteins Upon Admission Linked to COVID-19 Severity and Mortality? Front. Med. 8:718053. doi: 10.3389/fmed.2021.718053

\section{Are Reduced Levels of Coagulation Proteins Upon Admission Linked to COVID-19 Severity and Mortality?}

\author{
Francisco C. Ceballos ${ }^{1}$, Pablo Ryan ${ }^{2}$, Rafael Blancas ${ }^{3}$, María Martin-Vicente ${ }^{1}$, \\ Erick Joan Vidal-Alcántara ${ }^{1}$, Felipe Peréz-García ${ }^{4}$, Sofía Bartolomé ${ }^{1}$, \\ Juan Churruca-Sarasqueta ${ }^{5}$, Ana Virseda-Berdices ${ }^{1}$, Oscar Martínez-González ${ }^{3}$, \\ Oscar Brochado-Kith ${ }^{1}$, Marta Rava ${ }^{6}$, Carolina Vilches-Medkouri ${ }^{7}$, Natalia Blanca-López ${ }^{8}$, \\ Ignacio Ramirez Martinez-Acitores ${ }^{3}$, Patricia Moreira-Escriche ${ }^{9}$, Carmen De Juan ${ }^{9}$, \\ Salvador Resino ${ }^{1 *}$, Amanda Fernández-Rodríguez ${ }^{1 *+}$ and María Ángeles Jiménez-Sousa ${ }^{1 \dagger}$ \\ ${ }^{1}$ Unit of Viral Infection and Immunity, National Center for Microbiology (CNM), Health Institute Carlos III (ISCIII), Madrid, Spain, \\ ${ }^{2}$ Department of Infectious Diseases, Hospital Universitario Infanta Leonor, Madrid, Spain, ${ }^{3}$ Critical Care Department, Hospital \\ Universitario del Tajo, Aranjuez, Spain, ${ }^{4}$ Clinical Microbiology Department, Hospital Universitario Príncipe de Asturias, Alcalá \\ de Henares, Spain, ${ }^{5}$ Haematology and Haemostasis Department, University Hospital Infanta Leonor, Madrid, Spain, ${ }^{6}$ Unit \\ AIDS Research Network Cohort (CoRIS), National Center of Epidemiology (CNE), Health Institute Carlos III (ISCIII), Madrid, \\ Spain, ${ }^{7}$ Clinical Analysis Service, Hospital Universitario Príncipe de Asturias, Alcalá de Henares, Spain, ${ }^{8}$ Allergology \\ Department, University Hospital Infanta Leonor, Madrid, Spain, ${ }^{9}$ Department of Infectious Diseases, Hospital Universitario \\ Severo Ochoa, Madrid, Spain
}

Background: The link between coagulation system disorders and COVID-19 has not yet been fully elucidated.

Aim: Evaluating the association of non-previously reported coagulation proteins with COVID-19 severity and mortality.

Design: Cross-sectional study of 134 COVID-19 patients recruited at admission and classified according to the highest COVID-19 severity reached (asymptomatic/mild, moderate, or severe) and 16 healthy control individuals.

Methods: Coagulation proteins levels (antithrombin, prothrombin, factor_Xl, factor_XII, and factor_XIII) and CRP were measured in plasma by the ProcartaPlex Panel (Invitrogen) multiplex immunoassay upon diagnosis.

Results: We found higher levels of antithrombin, prothrombin, factor XI, factor XII, and factor XIII in asymptomatic/mild and moderate COVID-19 patients compared to healthy individuals. Interestingly, decreased levels of antithrombin and factors XI, XII, and XIII were observed in those patients who eventually developed severe illness. Additionally, survival models showed us that patients with lower levels of these coagulation proteins had an increased risk of death.

Conclusion: COVID-19 provokes early increments of some specific coagulation proteins in most patients. However, lower levels of these proteins at diagnosis might "paradoxically" imply a higher risk of progression to severe disease and COVID-19-related mortality.

Keywords: SARS-CoV-2, coagulation factors, COVID-19, mortality, SARS 


\section{HOW THIS FITS IN}

COVID-19 causes an early increase in coagulation proteins in most patients, even in those asymptomatic or with mild symptoms. Although not reflected in routine tests such as PT and aPTT, patients who lately advanced to severe disease, showed low levels of antithrombin, prothrombin, and factors XI, XII, and XIII at disease diagnosis. These reduced levels were associated for the first time with a higher COVID-19-related mortality.

\section{INTRODUCTION}

Coronavirus disease 2019 (COVID-19) is associated with a significant activation of the coagulation cascade. While thrombosis has been classically described in acute and chronic infections including respiratory diseases (1), thrombotic risk appears to be higher in COVID-19 (2). Consequently, thromboembolic complications are common in hospitalized patients, especially among those in intensive care units (ICUs) (3). In this sense, several mechanisms of coagulation activation have been postulated (4) and large dynamic fluctuations in coagulation and fibrinolysis laboratory parameters have been described during disease course (5). Development of overt disseminated intravascular coagulation (DIC) seems to be rare and to follow a different pattern from other infection-derived DIC (5-7), but it has been reported in up to $71 \%$ of fatal cases as a late and ominous sign (8).

Additionally, both venous and arterial thrombotic events have been independently associated with mortality (9). Several hemostatic-system abnormalities such as thrombocytopenia, elevated D-dimer levels, prolonged prothrombin time (PT) or activated partial thromboplastin time (aPTT), decreased factor $\mathrm{V}$ activity, hypofibrinogenemia, and reduced levels of natural anticoagulants (e.g., antithrombin) appear with increasing disease severity and have been linked to death $(6,8,10)$. However, the bidirectional relationship between SARS-CoV-2 and the coagulation system is still not completely understood (4). A predominant increase of $\mathrm{D}$-dimer is typical, and its presence on admission has been repeatedly described as significantly higher in non-survivors (11) but scarce or no abnormalities in PT and aPTT are usually found at disease onset $(5,12)$. To date, coagulation markers measured in the early phase of COVID19 have evidenced a complex scenario and elucidation of the pathophysiology of immunothrombosis is evolving. Therefore, to continue unraveling the insights of COVID-19-induced coagulopathy, we evaluate several coagulation proteins at an early stage of disease and their association with disease severity and mortality.

\section{METHODS}

\section{Design and Study Population}

In this cross-sectional study, 128 COVID-19 non-selected patients consecutively admitted to three different hospitals in Madrid (Infanta Leonor University Hospital, Aranjuez University Hospital, and Príncipe de Asturias University
Hospital), with an available plasma sample, were enrolled from March to September 2020.

Patients were classified according to their highest severity grade during the course of COVID-19 (Figure 1): (1) Severe: (i) death during hospitalization, (ii) ICU admission, (iii) invasive mechanical ventilation, or (iv) presence of bilateral pulmonary infiltrates, mechanical ventilation, and oxygen saturation $\left(\mathrm{Sat}_{2}\right)$ $\leq 93$. (2) Moderate: the remaining hospitalized patients who did not fulfill the severe COVID-19 criteria; (3) asymptomatic/mild (AM): individuals who had minor or no COVID-19 symptoms; (4) a control group of 16 pre-pandemic healthy controls without any known infection was included, they were age- and sexmatched with COVID-19 groups to limit confounding factors. The STROBE-ID checklist was used to strength the design and conduct the study.

\section{Clinical Data and Samples}

Epidemiological, clinical, and disease evolution data, as well as laboratory parameters such as PT, international normalized ratio (INR), and aPTT were collected from clinical records using an electronic case report form (eCRF) which was built using REDCap (13). Plasma samples were obtained after centrifugation of blood in EDTA tubes at hospital admission (median $=2$ days, $\mathrm{IQR}=4$ days). Samples were processed at the National Center for Microbiology (Majadahonda), Institute of Health Carlos III (Madrid, Spain).

\section{Coagulation Proteins}

Coagulation proteins (antithrombin, prothrombin, factor XI, factor XII, and factor XIII) and C-reactive protein (CRP) were measured by a Human Coagulation 6-PLEX ProcartaPlex Panel (Invitrogen), and D-dimer and IL-6 were measured by a multiplex immunoassay using a Bio-Plex $200^{\mathrm{TM}}$ system (Bio$\mathrm{Rad}$ ) according to the manufacturer's specifications. Coagulation proteins and their relationship in the coagulation cascade are shown in Figure 1.

\section{Statistical Analysis}

As outcome variables, the highest COVID-19 severity and mortality were considered. For descriptive data, differences between groups were tested using $\mathrm{Chi}^{2}$ or Fisher's exact test with a Monte Carlo-simulated $p$-value for categorical variables and Kruskal-Wallis test for continuous variables. The association between coagulation proteins, measured in the first days of disease, and the severity were explored using generalized linear mixed models (GLMMs). This analysis allows us to test the pairwise difference among disease severity classes by grouping the four coagulation proteins analyzed in this study. We fit a model where the protein was considered a random effect:

$$
\begin{aligned}
& Y_{\text {Severity class A, severity class B }}=\left(\beta_{0}+b_{p, 0 p}\right)+\beta_{1} X_{i} \\
& \beta_{2} X_{j}+\beta_{3} X_{k}+e_{p i j k}
\end{aligned}
$$

Where $\mathrm{bp}, 0 \mathrm{p}$ is the random effect of each coagulation protein, $\beta 1 \mathrm{Xi}$ is the fixed effect of the protein levels, $\beta 2 \mathrm{Xj}$ is the fixed effect of the age, and $\beta 3 \mathrm{Xk}$ is the fixed effect of the sex. By using this model, we tested whether we were able to 


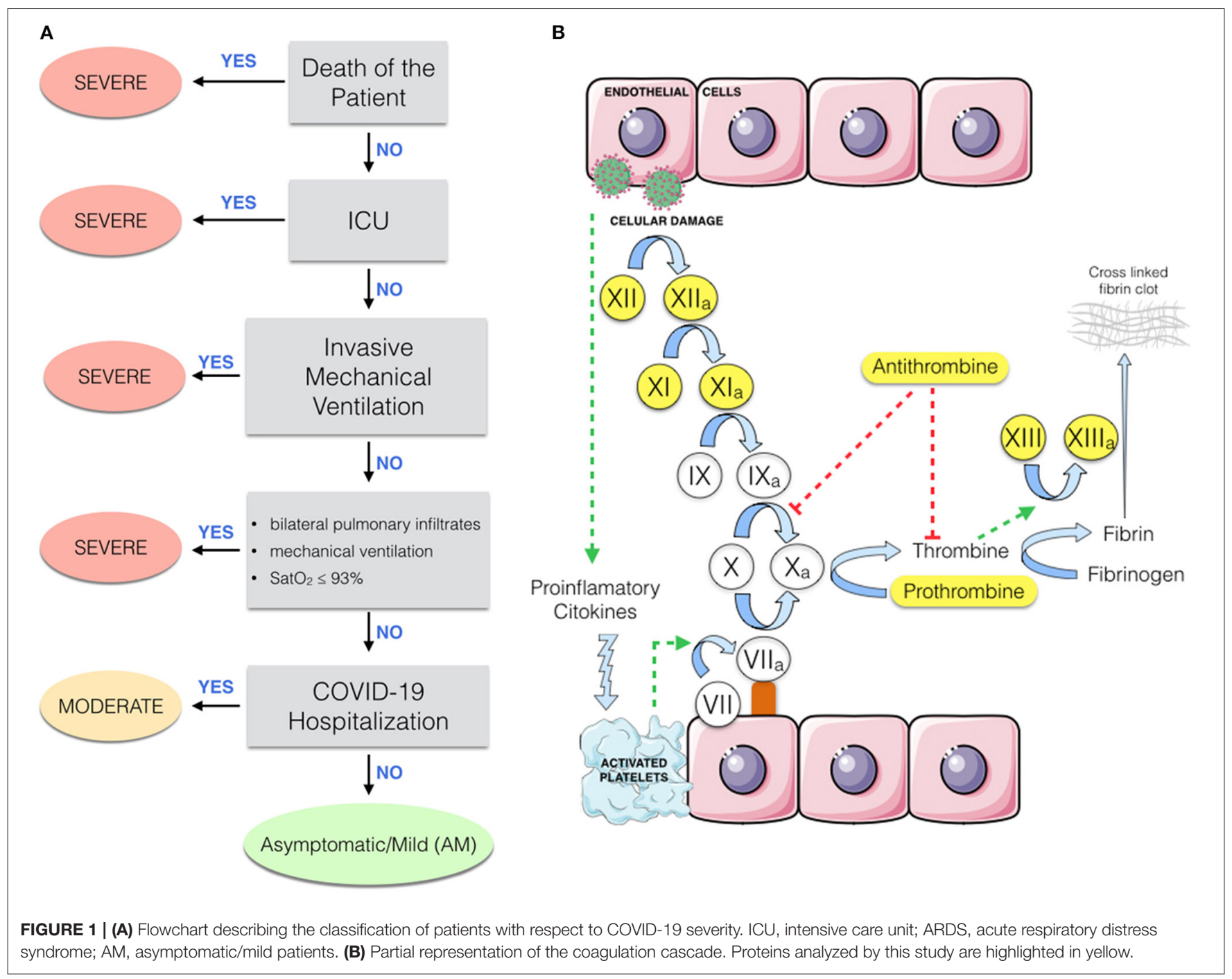

detect general effects between disease severity classes. We were also interested in studying the behavior of each coagulation protein. Pairwise comparisons between disease severity classes, for each protein, were carried out using multivariable logistic regressions. Sex and age were included as covariables in the multivariable analysis.

Survival time was defined as time between hospitalization and death, and individuals alive at 90 days were considered censored data. Survival curves were modeled using the Kaplan Meier method and a log rank test was performed to assess univariate differences in survival time according to coagulant proteins tertile levels. Death risk was estimated with the Cox proportionalhazard and Aalen's additive models. Age and sex were included as covariables. The survival Cox proportional-hazard model's goodness of fit was evaluated by the Harrel's concordance index (C-index). This index ranges from 0 to 1 and the intuition behind it is that, if the risk model is good, patients who had shorter timesto-death should have higher risk scores. Values of C-index near 0.5 indicate that the risk score predictions are not better than chance in determining which individual will die first. Coagulation proteins discrimination capabilities were measured by the area under the receiver operating characteristic (ROC) curve.

Two-sided tests were used for all statistical methods. Analyses were performed using the R 4.0.3 software.

\section{RESULTS}

\section{Clinical and Epidemiological Data of Patients}

Patient's characteristics are shown in Table 1. Coagulation proteins were measured before therapy administration, and no associations were found with comorbidities, chronic medications, or COVID-19 presentation (Supplementary Table 1; Supplementary Figure 1), showing no prior bias. Additionally, aPTT and PT (INR) did not show differences among the three groups of COVID-19 patients (Supplementary Figure 2). 
TABLE 1 | Patient's characteristics.

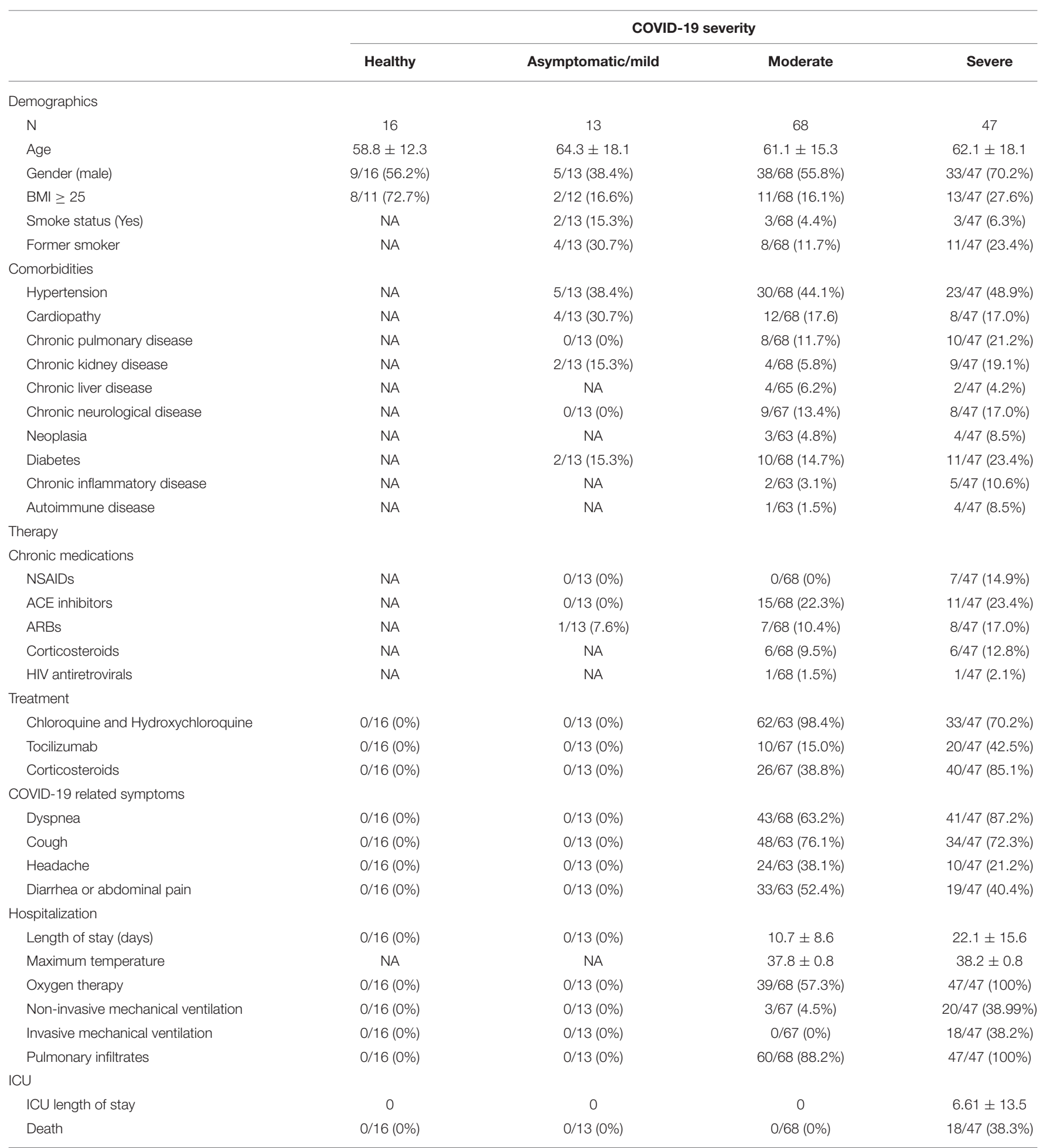

Patients were classified according to the highest disease severity reached during the COVID-19 evolution. Denominator indicates number of patients with available data. Statistics: Individual characteristics were summarized using standard descriptive statistics: mean \pm standard deviation for continue variables and count (percentage) for categorical variables. Differences between groups were tested using Fisher's exact test. No statistically significant association was found between covariates and disease severity with the exception of the following treatments: Chloroquine/hydroxychloroquine (Fisher's exact test $=1.6 e^{-05}$ ), Tocilizumab (Fisher's exact test $=8.1 e^{-05}$ ), corticosteroids (Fisher's exact test $=$ $5.7 e^{-08}$ ), and the use of supplemental oxygen (Fisher's exact test $=3.2 . e^{-07}$ ). NA, non-available; BMI, body mass index; NSAIDs, non-steroidal anti-inflammatory drugs; ACE, angiotensin-converting-enzyme; ARBs, angiotensin II inhibitors; HIV, human immunodeficiency virus; ICU, intensive care unit. 


\section{Coagulation Proteins Association With Severity}

Distribution of antithrombin, prothrombin, factor XI, factor XII, and factor XIII protein levels for each disease severity group is shown in Figure 2A. Statistical differences between severity groups are shown through generalized linear models in Figure 3. AM, moderate, and severe individuals had higher levels of coagulation proteins compared to healthy individuals. Also, we detected a significant reduction of factor XI, factor XII, and factor XIII levels in severe patients compared to moderate individuals (Supplementary Table 2). These pairwise differences between COVID-19 severity groups were also detected at the individual protein level (Figure 3; Supplementary Table 3).

Additionally, a traditional coagulation activation marker such as D-dimer and surrogate markers of inflammation such as CRP and IL-6 were also assessed. Statistical differences were observed mainly between moderate and severe groups, except for CRP, for which extreme groups showed the highest differences (Supplementary Table 4; Supplementary Figure 3). D-dimer showed significant correlation with prothrombin $\left(R^{2}=0.698, P=2.1 \mathrm{E}^{-22}\right)$ and factor XIII $\left(R^{2}=-0.216\right.$, $\left.P=3.7 \mathrm{E}^{-03}\right)$.

\section{Survival Analysis}

Kaplan-Meier analyses showed that patients with lower levels of the analyzed proteins had an increased risk of death during hospitalization (Figure 2B). For antithrombin, prothrombin, and factors XI and XIII, this increase was more accentuated in men (Supplementary Figure 4). The effect of proteins levels on survival was confirmed by the Cox proportional-hazard models and Aalen's additive regression (Supplementary Table 5). Both models found a significant effect of the different coagulation proteins on survival. These models found a negative correlation between the proteins' levels and the time of survival (Supplementary Table 5). A reduction in the coagulation proteins levels involves a higher risk of death, however, the effect size was slight for each individual protein (Supplementary Table 5). ROC curves showed that the addition of the coagulation proteins to the basic model composed by sex and age slightly improved the survival prediction although it was not significant (Supplementary Figure 5). We also performed survival analysis with aPTT and INR using different statistical models, but no significant association was observed (data not shown).

\section{DISCUSSION}

Our study shows that coagulation protein levels are affected at the first stages of COVID-19 and that these early changes already reflect disease severity. We report increased levels of antithrombin, prothrombin, factor XI, factor XII, and factor XIII in $\mathrm{AM}$ and moderate patients, compared to healthy individuals. In contrast, significantly decreased levels of antithrombin and factors XI, XII, and XIII were found at presentation in patients who followed a severe course with respect to moderate patients. By considering the coagulation protein as a random effect (in a logistic mixed model context), we could suggest a general activation of the coagulation cascade between healthy individuals and COVID-19 patients, and also a general decrease in protein levels in severe COVID-19 patients. As expected, D-dimer and IL-6 were significantly higher in severe patients than moderate patients. Moreover, CRP showed higher levels in moderate and severe patients than healthy and AM individuals. Additionally, aPTT and PT (INR) did not show differences among the three groups of COVID-19 severity, neither with survival. When we explored the effects of the coagulant proteins' levels on survival defined as time-to-death, we observed that patients with lower levels of the analyzed proteins had an increased risk of death during hospitalization.

Finally, we describe a sex-specific effect in further analyses, showing differences in the coagulation cascade regulation in both men and women. Variable sex was significant in the general mixed model and survival analysis. The negative association between coagulation proteins and disease severity was, in general, more pronounced in men. Our results seem to be in agreement with the clinical experience that found that men hospitalized with COVID-19 had more severe thrombosis than women (14).

\section{Strength and Limitations}

We present the evaluation of coagulation factors underrepresented in the literature as an additional approach to study coagulopathy in COVID-19 disease. In the results we report new associations of some protein factors with COVID-19 severity and include specific groups of patients with scarce coagulation data, such as asymptomatic patients. We also add healthy non-hospitalized individuals to address for collider bias (15). Furthermore, we were able to detect a sex-specific effect of SARS-CoV-2 on coagulation protein levels. Two main limitations should be considered. First, the limited sample size of healthy and asymptomatic cases, which could have limited the possibility of finding statistically significant differences for some comparisons. Secondly, we analyzed five coagulation proteins not routinely used in clinical practice; as coagulation cascade is extremely complex, further studies should consider additional factors to fully describe COVID-19 effects over the entire coagulation cascade. However, these additional factors have been extensively studied, and we analyzed those that were not previously addressed.

\section{Comparison With Existing Literature}

Since its worldwide outbreak in the first trimester of 2020, it has been systematically reported that COVID-19 is associated with a significant activation of the coagulation cascade. Our results are in accordance with previous studies (16) that show a consumption of coagulation proteins among COVID-19 nonsurvivors, or a reduction in abundance of prothrombin correlated with disease severity (17). Several recently published metaanalyses and reviews have shown significantly higher levels of D-dimer, fibrinogen, and fibrin in severe COVID-19 patients in comparison to non-severe (18-23).

However, as far as we know this is the first report studying coagulation factors XI, XII, and XIII in COVID-19, as most of the published studies have focused in other parameters such as 

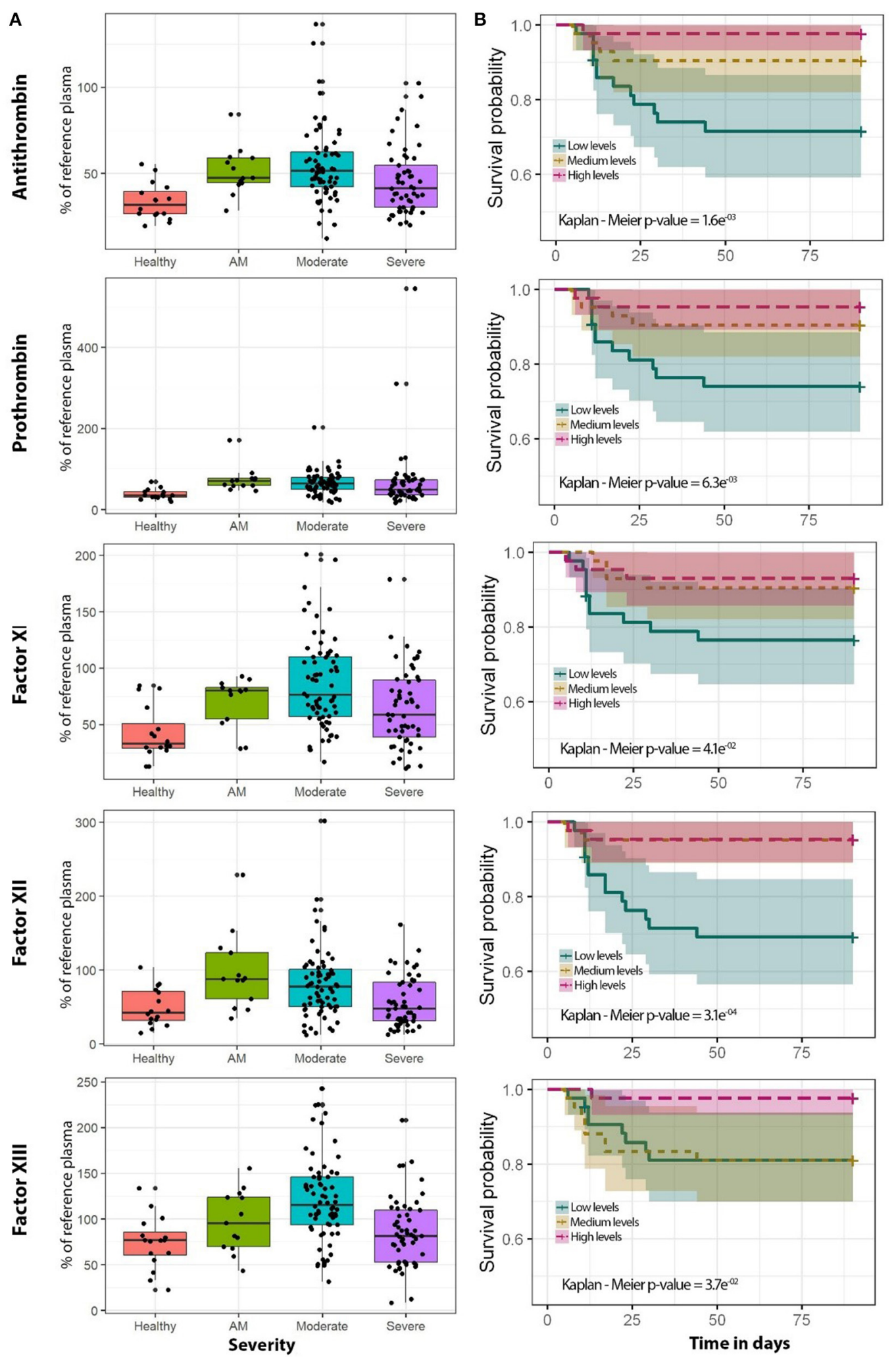

FIGURE 2 | Coagulation protein levels regarding COVID-19 severity and survival analysis for each of the coagulation proteins analyzed in this study. (A) Boxplots of healthy (purple, $n=16$ ), AM (blue, $n=13$ ), moderate (green, $n=68$ ), and severe (yellow, $n=47$ ) individuals. 
FIGURE 2 | (B) Kaplan-Meier plot. The cutoffs of coagulation proteins for the Kaplan-Meier plot were obtained using three quantiles to get low (blue), medium (yellow), and high (pink) percentages of the reference plasma. The specific categorical levels by using tertiles were as follows: Antithrombin: low (12.14-41.3), medium (41.3-54.1), high (54.1-136.7). Prothrombin: low (16.3-48.7), medium (48.7-72.2), high (72.2-545). Factor XI: low (10.8-56.4), medium (56.4-90.1), high (90.1-200.9). Factor XII: low (11.7-47.8), medium (47.8-87.1), high (87.1-301.9). Factor XIII: low (8.3-82.6), medium (82.6-118.3), high (118.3-242.7). AM, asymptomatic/mild patients; OLR, ordinal logistic regression.

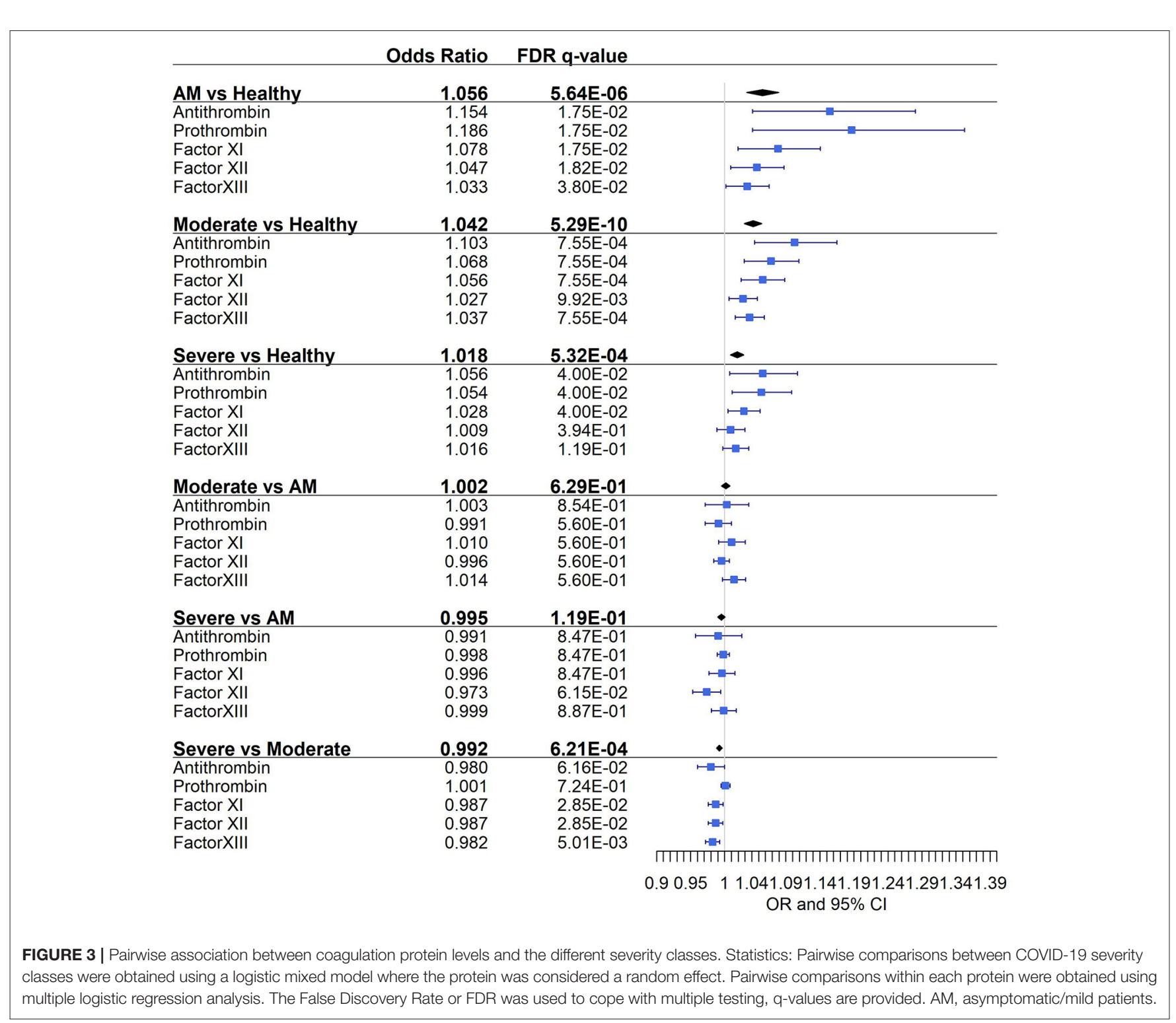

platelets, D-dimer, fibrin, fibrinogen, aPTT, PT, and even factor VIII and thrombin $(18,22,23)$.

The increase in natural anticoagulant and procoagulant proteins in COVID-19 has been attributed to the thromboinflammatory response caused by SARS-CoV-2, which provokes endotheliitis and increases the hepatic production of factors (24). This could explain the increases of clotting proteins found in our study even in early and mild stages.

Additionally, we showed that this negative association was more pronounced in men.
We observed that at the time of admission, there were no differences in aPTT and TP between survivors or non-survivors. These results are also in line with previous studies (2), where normal to slightly elevated aPTT or PT have been described in most COVID-19 patients at presentation. The INR, measured at the acute phase of the disease, seems to be elevated in nonsurvivors compared to survivors (8), however, no correlation was evident between COVID-19 severity and other DIC indicators like prolonged aPTT (16). Therefore, we suggest that an early reduced production or, more likely, an increased consumption 
(due to pulmonary or systemic coagulopathy) of clotting proteins could predict a worse prognosis.

The survival results were in accordance with previous studies (15) that show a consumption of coagulation proteins among COVID-19 non-survivors, however, we cannot rule out an impaired production of these coagulation proteins.

\section{Implications for Research and/or Practice}

In conclusion, our results indicate that: (1) COVID-19 causes an early increase of some specific coagulation proteins such as antithrombin, prothrombin, contact factors, and factor XIII in most patients, even in those who will not suffer from clinically significant disease, suggesting that commonly elevated D-dimer levels are driven by an initial enhanced procoagulant state and not just by hyperfibrinolysis; (2) although not reflected in routine tests such as PT and aPTT, and despite common initial hyperfibrinogenemia, patients who will eventually advance to severe disease show early decreased levels of these anticoagulant and procoagulant markers, suggesting either consumption or impaired production, and these levels were associated with higher COVID-19-related mortality. Evolving investigations will allow us to better clarify the crosstalk between the immune and clotting systems in this pandemic disease.

\section{DATA AVAILABILITY STATEMENT}

The raw data supporting the conclusions of this article will be made available by the authors, without undue reservation.

\section{ETHICS STATEMENT}

The studies involving human participants were reviewed and approved by the Ethics Committee of the Institute of Health Carlos III (PI 33_2020-v3) and the Ethics Committee of each hospital. The patients/participants provided their written informed consent to participate in this study.

\section{REFERENCES}

1. Grimnes G, Isaksen T, Tichelaar YIGV, Brækkan SK, Hansen JB. Acute infection as a trigger for incident venous thromboembolism: results from a population-based case-crossover study. Res Pract Thromb Haemost. (2018) 2:85-92. doi: 10.1002/rth2.12065

2. Hadid T, Kafri Z, Al-Katib A. Coagulation and anticoagulation in COVID-19. Blood Rev. (2020) 47:100761. doi: 10.1016/j.blre.2020.100761

3. Poissy J, Goutay J, Caplan M, Parmentier E, Duburcq T, Lassalle F, et al. Pulmonary embolism in patients with COVID19: awareness of an increased prevalence. Circulation. (2020) 142:184-6. doi: 10.1161/CIRCULATIONAHA.120.047430

4. Iba T, Connors JM, Levy JH. The coagulopathy, endotheliopathy, and vasculitis of COVID-19. Inflamm Res. (2020) 69:11819. doi: 10.1007/s00011-020-01401-6

5. Asakura H, Ogawa H. COVID-19-associated coagulopathy and disseminated intravascular coagulation. Int J Hematol. (2020) 7:1-13. doi: 10.1007/s12185-020-03029-y

6. Helms J, Tacquard C, Severac F, Leonard-Lorant I, Ohana M, Delabranche X, et al. High risk of thrombosis in patients with severe SARS-CoV-2 infection:

\section{AUTHOR CONTRIBUTIONS}

AF-R and MJ-S: funding body, supervision, and visualization. AF-R, MJ-S, and FC: study concept and design, statistical analysis, interpretation of data, and writing of the manuscript. PR, RB, MM-V, AV-B, OB-K, FC, FP-G, OM-G, CV-M, NB$\mathrm{L}$, and IR: patients' selection and clinical data acquisition. MM-V, AV-B, EV-A, SB, and OB-K: sample preparation and biomarker analysis. FC, AF-R, and MJ-S: writing of the manuscript. PR, RB, FP-G, JC-S, OM-G, MR, PM-E, $\mathrm{CD}$, and SR: critical revision of the manuscript for relevant intellectual content. All authors read and approved the final manuscript.

\section{FUNDING}

This study was supported by grants from Instituto de Salud Carlos III [ISCIII; Grant Number COV20/1144 (MPY224/20) to AF-R/MJ-S]. AF-R, MJ-S, and MR are Miguel Servet researchers supported and funded by ISCIII (Grant Numbers: CP14CIII/00010 to AF-R, CP17CIII/00007 to MJ-S, and CP19CIII/00002 to $\mathrm{MR})$.

\section{ACKNOWLEDGMENTS}

This study would not have been possible without the collaboration of all the patients, their families, medical and nursery staff, and data managers who have taken part in the project.

\section{SUPPLEMENTARY MATERIAL}

The Supplementary Material for this article can be found online at: https://www.frontiersin.org/articles/10.3389/fmed. 2021.718053/full\#supplementary-material

a multicenter prospective cohort study. Intensive Care Med. (2020) 46:108998. doi: 10.1007/s00134-020-06062-x

7. Muñoz-Rivas N, Abad-Motos A, Mestre-Gómez B, Sierra-Hidalgo F, CortinaCamarero C, Lorente-Ramos RM, et al. Systemic thrombosis in a large cohort of COVID-19 patients despite thromboprophylaxis: a retrospective study. Thromb Res. (2021) 199:132-42. doi: 10.1016/j.thromres.2020. 12.024

8. Tang N, Li D, Wang X, Sun Z. Abnormal coagulation parameters are associated with poor prognosis in patients with novel coronavirus pneumonia. J Thromb Haemost. (2020) 18:844-7. doi: 10.1111/jth.14768

9. Bilaloglu S, Aphinyanaphongs Y, Jones S, Iturrate E, Hochman J, Berger JS. Thrombosis in hospitalized patients with COVID-19 in a New York City Health System. JAMA. (2020) 324:799-801. doi: 10.1001/jama.2020.13372

10. Stefely JA, Christensen BB, Gogakos T, Cone Sullivan JK, Montgomery GG, Barranco JP, et al. Marked factor V activity elevation in severe COVID-19 is associated with venous thromboembolism. Am J Hematol. (2020) 95:152230. doi: 10.1002/ajh.25979

11. Zhang L, Yan X, Fan Q, Liu H, Liu X, Liu Z, et al. D-dimer levels on admission to predict in-hospital mortality in patients with Covid-19. J Thromb Haemost. (2020) 18:1324-9. doi: 10.1111/jth.14859 
12. Levi M, Iba T. COVID-19 coagulopathy: is it disseminated intravascular coagulation? Intern Emerg Med. (2020) 16:30912. doi: 10.1007/s11739-020-02601-y

13. Harris PA, Taylor R, Minor BL, Elliott V, Fernandez M, O’Neal $\mathrm{L}$, et al. The REDCap consortium: building an international community of software platform partners. J Biomed Inform. (2019) 95:103208. doi: 10.1016/j.jbi.2019.103208

14. Wilcox T, Smilowitz N, Berger J. Age and sex differences in incident thrombosis in patients hospitalized with COVID 19. J Am Coll Cardiol. (2021) 77:1826. doi: 10.1016/S0735-1097(21)03182-X

15. Griffith GJ, Morris TT, Tudball MJ, Herbert A, Mancano G, Pike L, et al. Collider bias undermines our understanding of COVID-19 disease risk and severity. Nat Commun. (2020) 11:5749. doi: 10.1038/s41467-020-19478-2

16. Martín-Rojas RM, Pérez-Rus G, Delgado-Pinos VE, Domingo-González A, Regalado-Artamendi I, Alba-Urdiales N, et al. COVID-19 coagulopathy: an in-depth analysis of the coagulation system. Eur J Haematol. (2020) 105:74150. doi: 10.1111/ejh.13501

17. Overmyer KA, Shishkova E, Miller IJ, Balnis J, Bernstein MN, Peters-Clarke TM, et al. Large-scale multi-omic analysis of COVID-19 severity. Cell Syst. (2021) 12:23-40.e7. doi: 10.1016/j.cels.2020.10.003

18. Lin J, Yan H, Chen H, He C, Lin C, He H, et al. COVID-19 and coagulation dysfunction in adults: a systematic review and meta-analysis. J Med Virol. (2021) 93:934-44. doi: 10.1002/jmv.26346

19. Xiang G, Hao S, Fu C, Hu W, Xie L, Wu Q, et al. The effect of coagulation factors in 2019 novel coronavirus patients: a systematic review and metaanalysis. Medicine. (2021) 100:e24537. doi: 10.1097/MD.0000000000024537

20. Rahi MS, Jindal V, Reyes SP, Gunasekaran K, Gupta R, Jaiyesimi I. Hematologic disorders associated with COVID-19: a review. Ann Hematol. (2021) 100:309-20. doi: 10.1007/s00277-020-04366-y

21. Gu SX, Tyagi T, Jain K, Gu VW, Lee SH, Hwa JM, et al. Thrombocytopathy and endotheliopathy: crucial contributors to COVID-19 thromboinflammation. Nat Rev Cardiol. (2021) 18:194-209. doi: 10.1038/s41569-020-00469-1
22. Ortega-Paz L, Capodanno D, Montalescot G, Angiolillo DJ. Coronavirus disease 2019-associated thrombosis and coagulopathy: review of the pathophysiological characteristics and implications for antithrombotic management. J Am Heart Assoc. (2021) 10:e019650. doi: 10.1161/JAHA.120.019650

23. Lazzaroni MG, Piantoni S, Masneri S, Garrafa E, Martini G, Tincani A, et al. Coagulation dysfunction in COVID-19: the interplay between inflammation, viral infection and the coagulation system. Blood Rev. (2021) 46:100745. doi: 10.1016/j.blre.2020.100745

24. Wise J. Covid-19 and thrombosis: what do we know about the risks and treatment? BMJ. (2020) 369:m2058. doi: 10.1136/bmj.m2058

Conflict of Interest: The authors declare that the research was conducted in the absence of any commercial or financial relationships that could be construed as a potential conflict of interest.

Publisher's Note: All claims expressed in this article are solely those of the authors and do not necessarily represent those of their affiliated organizations, or those of the publisher, the editors and the reviewers. Any product that may be evaluated in this article, or claim that may be made by its manufacturer, is not guaranteed or endorsed by the publisher.

Copyright (C) 2021 Ceballos, Ryan, Blancas, Martin-Vicente, Vidal-Alcántara, PerézGarcía, Bartolomé, Churruca-Sarasqueta, Virseda-Berdices, Martínez-González, Brochado-Kith, Rava, Vilches-Medkouri, Blanca-López, Ramirez Martinez-Acitores, Moreira-Escriche, De Juan, Resino, Fernández-Rodríguez and Jiménez-Sousa. This is an open-access article distributed under the terms of the Creative Commons Attribution License (CC BY). The use, distribution or reproduction in other forums is permitted, provided the original author(s) and the copyright owner(s) are credited and that the original publication in this journal is cited, in accordance with accepted academic practice. No use, distribution or reproduction is permitted which does not comply with these terms. 